\title{
LA NECESIDAD Y LA OPORTUNIDAD DE LA INNOVACIÓN
}

\section{THE NEED AND OPPORTUNITY OF INNOVATION}

Mónica García Cantó ${ }^{1}$

Laura Ma Palacios Ruiz²

1. Consultora especialista en el área de innovación y financiación. Executive Máster en Innovación (EOI). Ingeniera de Organización Industrial e Ingeniera Técnica especializada en Química por la Universidad de Valencia (EPSA).E-mail: monigc22@hotmail.com

2. Consultora especialista en el área de innovación y organización industrial. Grado de Ingeniería Civil e Ingeniería Técnica de Obras Públicas. Máster Universitario en Organización Industrial y Logística por la Universidad de Valencia (EPSA). E-mail: Impalaciosr@gmail.com

\section{Citación sugerida:}

García Cantó, M. y Palacios Ruiz, L.M. (2017). La necesidad y la oportunidad de la innovación. $3 C$ Tecnología: glosas de innovación aplicadas a la pyme, 6(1), 47-52. DOI: <http://dx.doi.org/10.17993/3ctecno.2017.v6n1e21.47-52/>. 


\section{RESUMEN}

Se realiza un análisis de la necesidad y oportunidad de la innovación como opción de supervivencia. Así mismo, se estudian los diferentes conceptos de innovación, tecnología y su importancia para el desarrollo empresarial, se exponen sus fases y alcance. Se realiza un análisis pormenorizado de las dificultades de la función innovación en la pyme. Se incorpora una descripción de aquellas herramientas consideradas básicas para poder gestionar con éxito la innovación, acabando con un apartado de conclusiones.

\section{ABSTRACT}

In this research, an analysis of the necessity and opportunity of the innovation as an option of survival is realized. Besides, the different concepts of innovation, technology and its importance for the enterprise development are studied, its phases and reach are exposed. It is performed a detailed analysis of the difficulties of innovation in SMEs function. It is incorpored a description of those basic tools to successfully manage innovation, ending with a section of conclusions.

\section{PALABRAS CLAVE}

Innovación tecnológica, investigación y desarrollo, gestión de la innovación, herramientas para la innovación.

\section{KEY WORDS}

Technological innovation, research and development, management of innovation, tools for innovation. 


\section{INTRODUCCIÓN}

La historia nos ha revelado en reiteradas ocasiones que la mayoría de las innovaciones aparecen ante períodos críticos que desencadenan problemáticas que necesitan ser resueltas de forma efectiva.

En estos momentos de crisis, en los que la economía española y europea se encuentran debilitadas y disminuidas, aparece el factor o función innovación como elemento que jugará un papel fundamental en su reactivación.

Por otra parte, el entorno empresarial, la globalización y la extraordinaria capacidad de la sociedad para crear conocimiento abren un enorme abanico de oportunidades para innovar. En cuanto a la sociedad, cada vez más exigente, impulsa cambios en el entorno empresarial próximo, siendo una fuente constante de retos que inducen cambios en la oferta de las empresas y en la forma de llevarla al mercado.

La globalización convierte en accesibles mercados algunos antes descartados, así como abre también los habituales a nuevos competidores que pueden tener otras ventajas. Sólo con cambios, es decir, con innovaciones, será posible hacer frente a las nuevas situaciones, sean estas oportunidades 0 amenazas para la empresa.

Estas son sólo algunas de las razones que hacen que las innovaciones sean las que marcan el camino para asegurar la permanencia de la empresa y para aprovechar oportunidades que distingan su oferta.

\section{2. ¿QUÉ ES LA INNOVACIÓN Y EN QUÉ CONSISTE?}

En ocasiones se asocia la innovación exclusivamente con la Investigación y Desarrollo, I+D. Si bien el factor tecnológico está presente en gran parte de la actividad innovadora de las empresas, la función innovación incluye otras actividades no necesariamente basadas en la tecnología.

Desde un punto de vista más amplio, podríamos definir la Innovación como el arte de convertir las ideas y el conocimiento en productos, procesos o servicios nuevos, significativamente mejores que los existentes, y sobre todo, así sean valorados o reconocidos por el mercado, sin excluir nuevos enfoques de marketing, nuevas formas de distribución, cambios en la organización,...

Por lo tanto, Innovación no solo es añadir mayor sofisticación técnica o tecnológica a los productos o servicios, sino que estos se adapten mejor a las necesidades del cliente o usuario final. Así pues, los tres principios básicos que debe cumplir toda innovación o desarrollo son fundamentalmente tres [1], [2], [3]:

1. Debe cubrir alguna necesidad, ser útil.

2. Se tiene que poder repetir y continuar con ello.

3. Debe poder ser asumida y gestionada y soportada por la organización o empresa que la desarrolla.

Aunque existen diferentes tipos de clasificaciones de la innovación, la mayoría suelen basarse en el siguiente esquema [1], [3], [4]: 


\section{$\left.{ }^{3} \mathrm{c}\right)$ tecnología}

3C Tecnología (Edición 21) Vol.6 - № 1

Marzo - junio'17, 47-52

Área de Innovación y Desarrollo, S.L.

ISSN: $2254-4143$

DOI: http://dx.doi.org/10.17993/3ctecno.2017.v6n1e21.47-52

- Innovación tecnológica.

- Innovación de producto.

- Innovación de servicio.

- Innovación de proceso.

- Innovación en la gestión de las relaciones con el cliente.

- Innovación organizacional.

Desde un punto de vista estratégico, la innovación no es una alternativa de negocio, tampoco es solo cosa de empresas con mucho nombre y tamaño, es una necesidad, quizás la única opción de supervivencia para las pymes, dado que la competencia de mercado global no nos permite hacer las cosas como hasta ahora.

Desde esta perspectiva la innovación en la pyme será aquello que nos da la oportunidad de diferenciarnos, ser muy buenos en algunos aspectos en los que otras empresas no operan.

\section{DIFICULTADES EN LA GESTIÓN DE LA INNOVACIÓN EN LA PYME}

En general, la dificultad real para la adopción de innovaciones no está en la propia innovación, sino en su implantación práctica, siendo necesario contemplar los siguientes factores clave para que una innovación se implante con éxito [4], [6]:

- Falta de tiempo. La gerencia y directivos de la empresa no tienen tiempo para desarrollar con éxito la función innovación.

- No adecuación de la estructura de la empresa. Las estructuras verticales, departamentales o jerárquicas conllevan falta de fluidez de los procesos, caracterizándose por ser "reinos de taifas" o compartimentos estancos, mientras que las estructuras horizontales o por proyectos conllevan la no dependencia temporal de los participantes, mientras dura el proyecto, de sus departamentos o secciones. Las estructuras matriciales son sin ninguna duda una solución óptima, aunque no es fácil ni inmediata su diseño e implantación.

- Inexistencia o no adecuación de la estructura organizativa de la innovación. Comité de innovación, director de proyecto, director funcional, administrador del proyecto, ...

- Carencias formativas, relativas a los mecanismos y herramientas para innovar, del personal clave de la empresa. La orientación hacia el aprendizaje es necesaria para que los colaboradores estén dispuestos a experimentar y a asumir los riesgos de aprender algo nuevo.

- Falta de cultura, incentivación, motivación hacia la innovación. La percepción de los miembros del equipo sobre la importancia y necesidad de implantar la innovación, lo que supone una resistencia al cambio y una visión cortoplacista,

- Enorme distanciamiento enorme entre universidades, centros de investigación y empresas. 


\section{HERRAMIENTAS BÁSICAS PARA LA GESTIÓN DE LA INNOVACIÓN}

En este apartado pretendemos enumerar y realizar una pequeña descripción de las metodologías y herramientas que deben ser puestas en práctica por una organización de cualquier tipo o tamaño para implementar con éxito la función innovación [5], [6], [7].

- Técnicas de creatividad que permiten el entrenamiento creativo. Como se fomentan las ideas y como éstas se convierten en innovación.

- Evaluación y selección de las ideas o proyectos. Análisis de viabilidad técnica y de mercado.

- Estado del arte, estudio de la técnica, antecedentes a la innovación.

- Vigilancia tecnológica e inteligencia competitiva como proceso organizado, selectivo y permanente de captar información del exterior y de la propia organización sobre ciencia, tecnología y mercados, seleccionarla, analizarla, difundirla y comunicarla, para convertirla en conocimiento para tomar decisiones con menor riesgo y poder anticiparse a los cambios

- Generación y selección de proyectos. Creación del mapa de proyectos, indicación de dificultad, impactos, costes, recursos, etc. con indicación de zonas de ausencia, análisis y diversificación de riesgos.

- Selección, propiamente dicha, a partir de los proyectos presentados según los pasos anteriores.

- Especificación, planificación, control y seguimiento de proyectos: determinación y control de objetivos, hitos, actividades, recursos necesarios, responsabilidades, entregables, tiempos,...

- Revisión y cierre de proyectos: cierre administrativo, cuentas, contratos con proveedores, recogida y archivo de información. Se incluirá en esta fase la revisión y extracción de conclusiones para el desarrollo de nuevos proyectos, en base a la consecución de los objetivos, el cumplimiento de los plazos, el uso de recursos, costes, etc.

\section{CONCLUSIONES}

Si bien es cierto que la innovación es fundamental para el éxito y supervivencia de las organizaciones, también es cierto que la mayoría de gerentes y directores no están satisfechos con la capacidad innovadora de sus empresas.

Se equivocan aquellos que entienden la "innovación" como un factor espontaneo, no estructurado, ligado a la "prueba y error", suerte o azar. La innovación, como cualquier otra función y subsistema de la empresa, es sistemática, está basada en el conocimiento y solo tendrá éxito si tiene una orientación de proceso, si se la destina de recursos suficientes y de herramientas y mecanismos específicos que garanticen su consecución. 


\section{REFERENCIAS BIBLIOGRÁFICAS}

[1] Florángel Ortiz. (2006). Gestión de innovación tecnológica en PYMES manufactureras. México.

[2] Cluster Audiovisual Galego (2011). El sistema de gestión de la innovación. Milladoiro, A Coruña.

[3] Martínez García, F.J. (2007). Estrategia e innovación de la pyme industrial en la comunidad de Cantabria, Santander.

[4] Robotiker. (2001). Guía Tecnopyme.

[5] CEM. (2009). Guía práctica de innovación para pymes.

[6] Ramis Pujol, J. (2009). Guía práctica de la innovación para PYMES.

[7] Fundación Cotec. (2010). La innovación en un sentido amplio. Análisis conceptual y empírico. 